\title{
Greater Nursing Role for Enhanced Post-Percutaneous Coronary Intervention Management
}

\author{
Tian Zhang' \\ Xiangxiu $\mathrm{Qi}^{2}$ \\ 'The First Internal Medicine \\ Comprehensive Ward of Shengjing \\ Hospital, China Medical University, \\ Shenyang, People's Republic of China; \\ ${ }^{2}$ Nursing Department of Shengjing \\ Hospital, China Medical University, \\ Shenyang, People's Republic of China
}

\begin{abstract}
Percutaneous coronary intervention (PCI) has increasingly been used in management of coronary artery diseases (CAD). Coupled that with an increasing incidence rate of $\mathrm{CAD}$ has augmented the hospital burden with consequential post-PCI patient management problems and dissatisfaction. Nursing care has a key role to play in upgrading the healthcare services and raising patients' satisfaction through enhanced patient education and engagement. In this regard, nursing-led intervention has shown some success in three main domains: risk reduction; psychological improvement; and quality of life. Urgent efforts are needed to formulize a structured follow-up with enhanced nursing role in post-PCI management to raise quality of healthcare.
\end{abstract}

Keywords: ischemic heart disease, nursing care, healthcare services, quality of life

\section{Coronary Artery Disease (CAD)}

Coronary artery disease (CAD), also known as ischemic heart disease (IHD) and atherosclerotic cardiovascular disease (ACD), is a leading cause of death worldwide. ${ }^{1}$ CAD is clinically manifested as myocardial infarction (MI) and ischemic cardiomyopathy with atherosclerosis as the main underlying pathological process. ${ }^{2,3}$ Incidence of CAD may continue to rise due to significant increase in prevalence of main associated factors or trends such as smoking, diabetes, obesity, metabolic syndromes, aging, and behavioral changes associated with urbanization and globalization. ${ }^{4-6}$ Current epidemiological data on IHD from the Global Burden of Disease (GBD) is illustrated in Figure $1 .^{7}$

\section{Percutaneous Coronary Intervention (PCI) for CAD}

PCI in the form of balloon angioplasty and/or stent implantation has been increasingly used as the initial management strategy with a clear advantage over optimal medical therapy [OMT] in terms of reducing mortality and myocardial infarction (MI). ${ }^{8-11}$ As a result, increased admissions/readmissions, hospitalizations, and discharge procedures, as well as, hospital cost in terms of labor and equipment has risen. ${ }^{12,13}$ To address this issue, same-day discharge is evaluated and considered viable and safe and is also favored by most patients and family members. ${ }^{14-16}$ Nonetheless, certain issues have evolved and remains to be addressed as illustrated in Figure 2C. Correspondence: Xiangxiu Qi
Nursing Department of Shengjing Hospital, China Medical University, No. 36 Sanhao Street, Heping District, Shenyang, Liaoning Province, I 10004 People's Republic of China Email 492215122@qq.com 
A

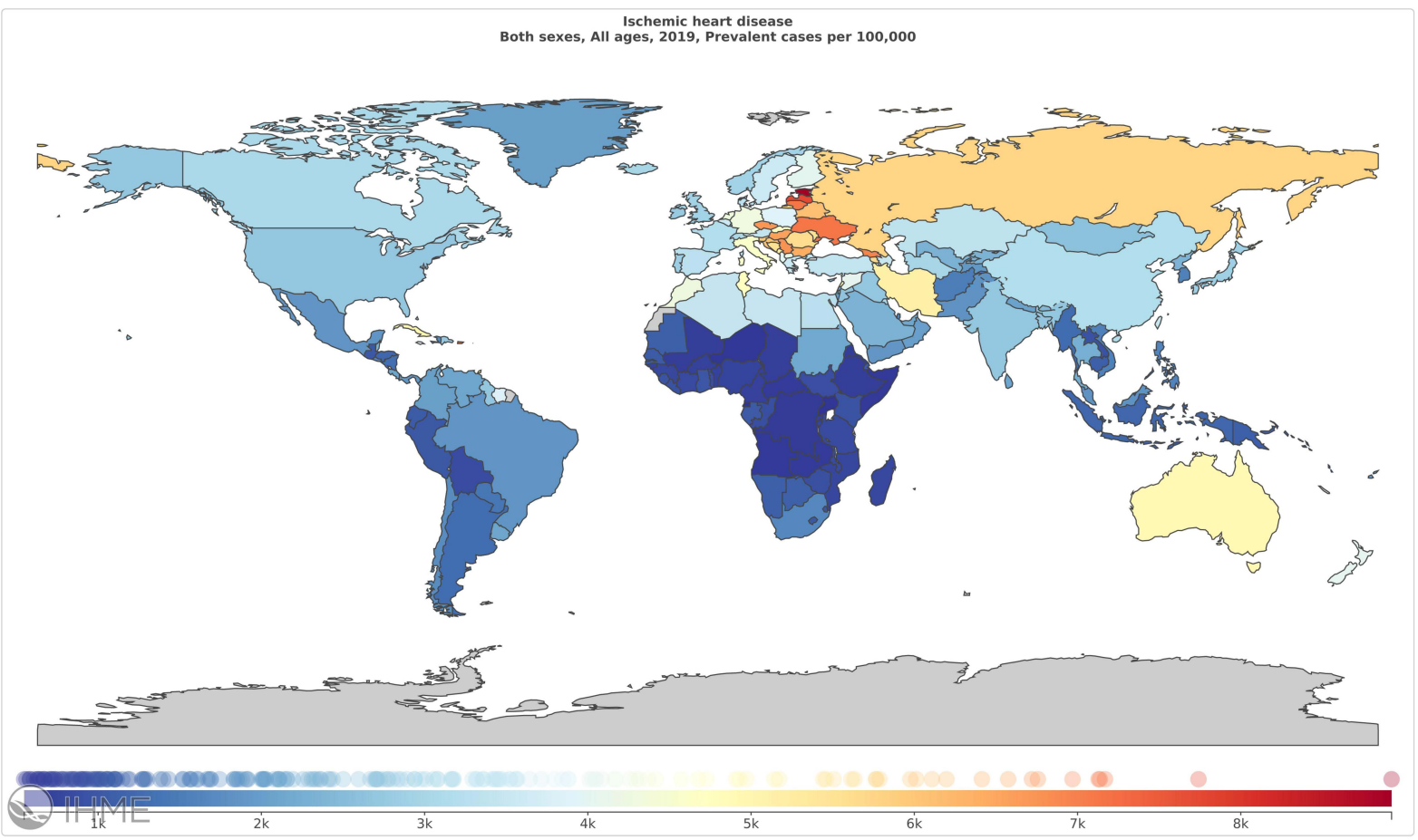

B

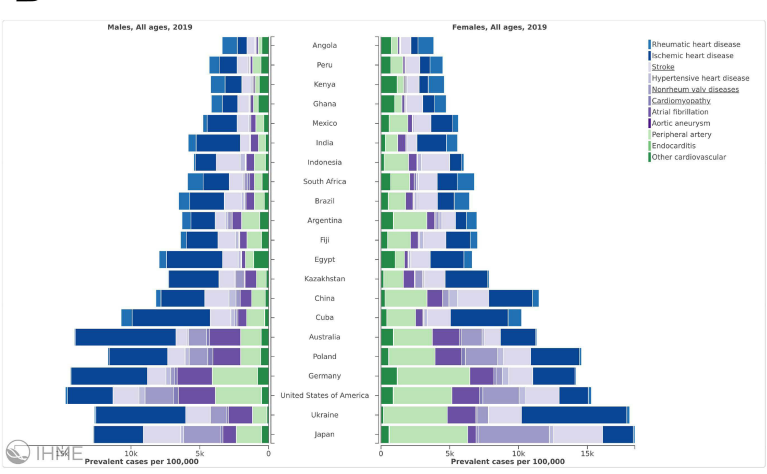

C

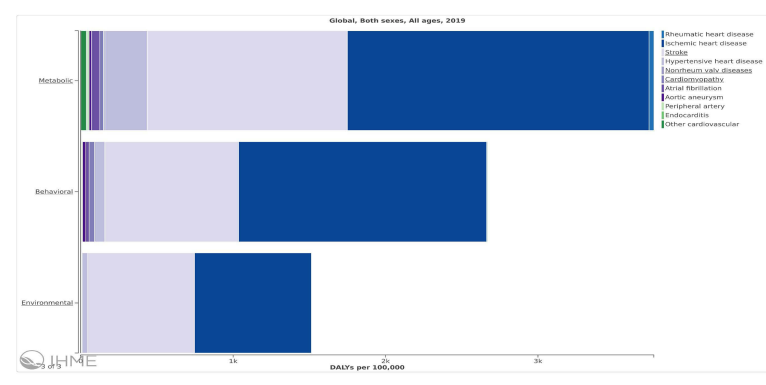

D

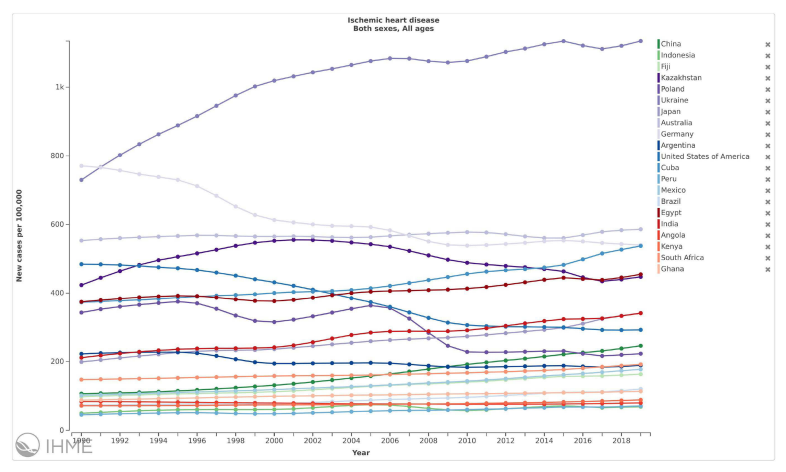

E

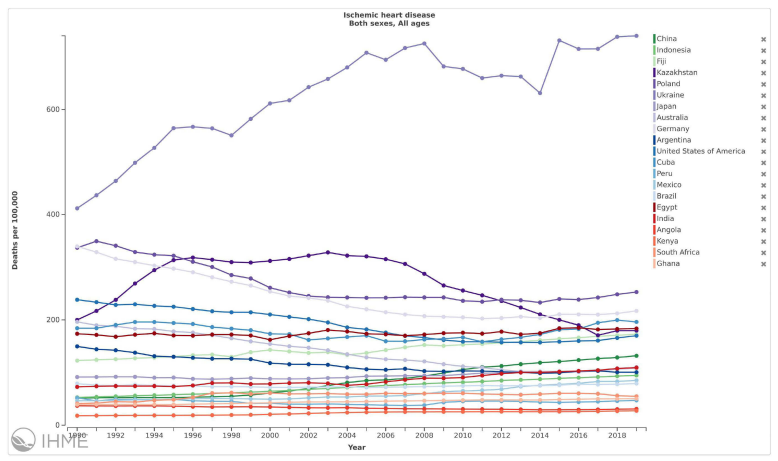

Figure I Current epidemiological data on ischemic heart disease (IHD) from the Global Burden of Disease (GBD). (A) Global prevalence of ischemic heart disease (colors indicate prevalence rates per 100,000 population in 2019). (B) Prevalence of IHD among cardiovascular diseases in males and females in various countries. (C) Association of risk factors, including metabolic, behavioral, and environmental, and IHD. (D) Incidence trend from 1990 to 2018 according to countries. (E) Global mortality trends. Notes: Current epidemiological data on IHD was obtained from Institute for Health Metrics and Evaluation (IHME). The Global Burden of Disease (GBD) dataset. Available from: http://vizhub.healthdata.org/gbd-compare. Used with permission. All rights reserved. ${ }^{7}$ 


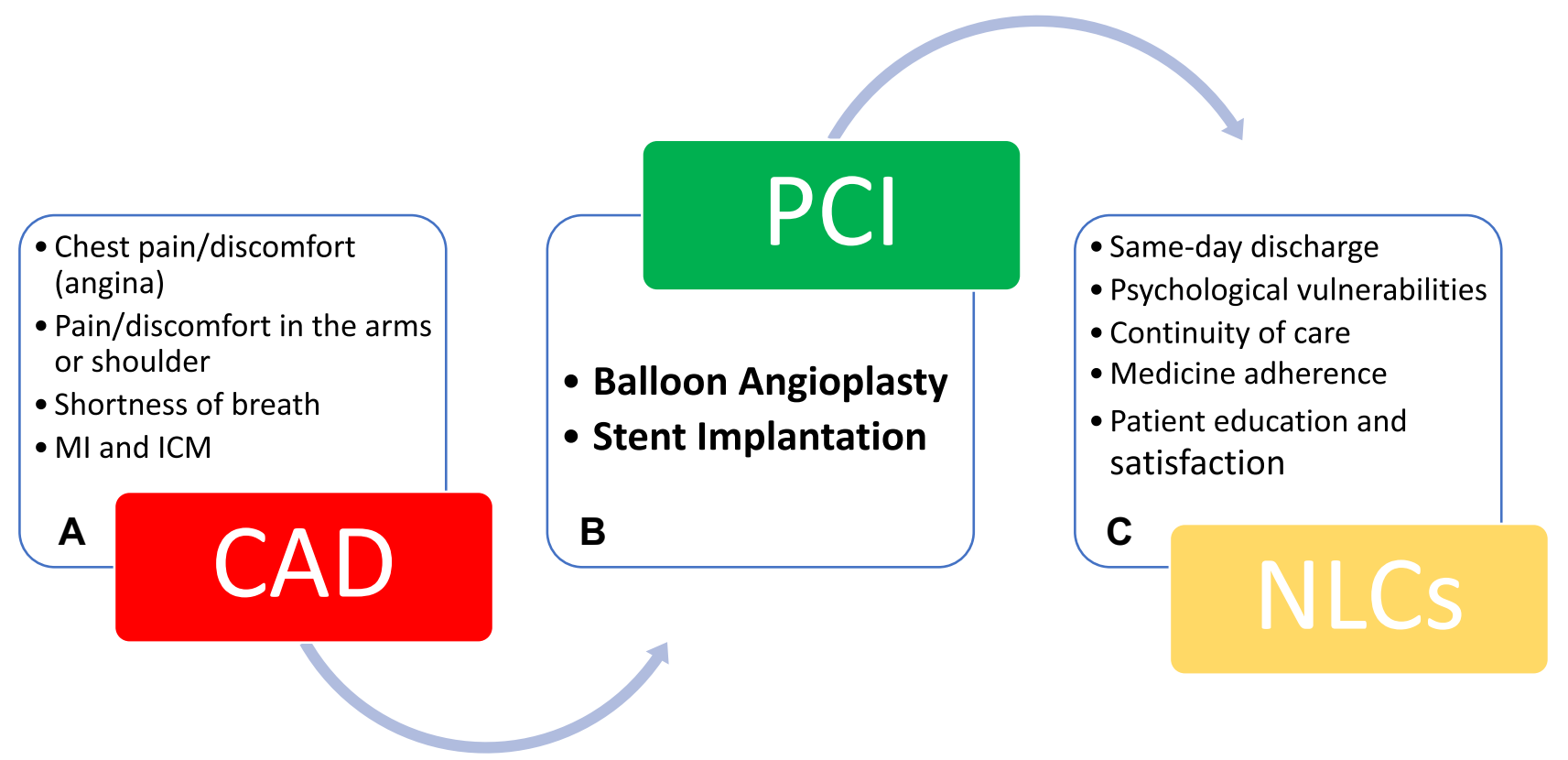

Figure 2 Nursing role in post-PCl management. (A) Coronary artery disease is manifested by pain or discomfort in chest (angina) and/or in the arms and shoulders with shortness of breath. (B) Percutaneous coronary intervention (PCl) is undertaken in the form of balloon angioplasty or stent implantation to relieve symptoms. (C) Post-PCl management has revealed several problems that requires enhanced nursing role.

Abbreviations: CAD, coronary artery disease; MI, myocardial infarction; ICM, ischemic cardiomyopathy; PCl, percutaneous coronary intervention; NLCs, nursing led clinics.

\section{Post-PCI Conundrums}

Patients undergoing PCI have encountered an upsurge in psychological burden and deteriorated quality of life due to lack of information and education about post-discharge health management plan, referral for secondary preventions, self-care compliance, and understanding of their chronic condition. ${ }^{15,17-21}$ Vulnerability and anxiety were revealed for lack of post-discharge instruction and uncertainty in assessment of same-day discharge following PCI. ${ }^{15}$ In certain cases, uncertainty about their future health and fear for disease recurrence has led to motivation for adopting a healthy lifestyle. While perception of being cured without realization of chronic health condition has resulted in decreased engagement in risk reduction behaviors. ${ }^{18}$ Continuity of care following PCI has also been a challenge. An unplanned journey across care boundaries was observed as a result of patients' failure to integrate health information due to lack of adequate instruction and information. Furthermore, facilitation was also required for connections to community-based resources and to schedule clear follow-up appointments. ${ }^{19}$ Following first-time PCI, some patients have been found critical of physicians and nurses for uninformative and inadequate medication information for failure to adhere to a medication regimen. While others have reasoned side effects, generic drugs skepticism, lack of information about disease seriousness, and psychological impact of living with CAD. ${ }^{20}$ Readmissions in the early post-discharge period are common and repeatedly linked to limited access to self-care education and preparation. $^{21}$ To summarize, enhanced education and information related to post-PCI healthcare management, symptoms attribution, medicine adherence, and readmission assistance should be of prime importance in order to improve community health services.

\section{Greater Nursing Role and Nursing-Led Clinics (NLCs)}

Rapidly expanding medical needs of individuals, communities and healthcare services asks for a broader role and scope of nursing practice. Nursing care has evolved over the years from primary healthcare nursing to nursing-led clinics in modern days. Here, we will review clinical evidence of efficacy of the nursing care in aforementioned scenarios. Management of post-MI/PCI patients can benefit a lot from lifestyle changes and risk reduction behaviors. Nurses can play a key role in education and care that can support and promote patient's engagement in these risk reduction activities. ${ }^{18}$ Nursing intervention leading to significant 
improvement in lifestyle changes were observed in an RCT, initiated by Lindsay et al, involving 95 (intervention $=45$; control=50) first-time percutaneous transluminal coronary angioplasty (PTCA) patients. These improvements included significant increase in exercise participation and greater adherence to a healthy heart diet in the intervention cohort at 6 weeks $(p=0.04$; $p=0.044)$ and 6 months $(p=0.05 ; p=0.035)$, respectively. Moreover, four of the five smoker patients had either quit or actively pursuing to stop smoking at 6 months. ${ }^{22}$ Similarly, in a quasi-experimental trial of 30 newly diagnosed ACS patients with PCI intervention, significant difference was detected in self-care compliance including smoking cessation. ${ }^{23}$ Smoking cessation was also an apparent feature of Corones-Watkins et al pilot RCT with 17 patients positive for smoking history had reported of smoking cessation at all time points (days 57, 1 month, 3 months). ${ }^{24}$ Moreover, a moderate reduction in cardiac self-efficacy (CSE) in the intervention group $(\mathrm{p}=0.60)$ at days $5-7$ and 1 month was also achieved. $^{24}$ Hence, medical literature review suggest that a greater nursing role is feasible and certainly helpful in educating and encouraging patients about selfmanagement and undertaking as well as adhering to risk reduction lifestyle changes.

Another aspect that certainly requires greater nursing intervention is the psychological and mental well-being. In fact, nurse-led psychological intervention has shown to reduce anxiety and improved quality of life in post-PCI patients. In a randomized controlled trial of 80 patients (intervention $=40$; control=40), significantly higher anxiety level, which was assessed with Zung Self-Rating Anxiety Scale (SAS), in the control group was observed at 12 months follow-up than the baseline and compared to intervention group. $^{25}$ Mean trait anxiety score assessed with State-Trait Anxiety Inventory (STAI), which is a psychological inventory based on a 4-point Likert scale and consists of 40 questions on a self-report basis, had shown greater decrease in the intervention group than in the control group at days 5-7 and 1 month in the randomized pilot study of nurse-led clinic initiated by CoronesWatkins et al. ${ }^{24}$ Intervention group also had achieved greater total change $(d=0.50)$ from baseline than in the control group $(\mathrm{d}=0.16) .{ }^{24}$ Depressive symptoms assessed with Cardiac Depression Scale (CDS) were also reduced in the intervention $(d=0.26)$ as well as control group $(d=0.37)$. These studies indicate that nurse-led initiative can successfully lower the anxiety level in post-PCI patients thereby lowering the psychological burden which may also yield long-term benefits for these patients.

Nursing intervention have also shown to improve quality of life assessed with various clinical indices. In a randomized controlled trial $(n=80)$, significant progress was seen in intervention group assessed with three domains of Seattle Angina Questionnaire (SAQ) 12 months after undergoing PCI. ${ }^{25}$ In the Lindsay et al. RTC, quality of life assessed with Quality-of-Life IndexCardiac Version III (QLI-CVIII) showed significant difference between the groups for QLI-CVIII scores at 6 weeks $(p=0.05)$ and 6 months $(p=0.04)$, but not at 1 year. ${ }^{22}$ Shim and Hwang also reported improvement in mental health assessed with scale based on American Heart Association Cardiovascular Risk Factor Assessment Tool Health-related quality of life (HRQoL). Overall, nursing-led intervention has demonstrated improvement in three main dimensions of post-PCI scenario in short term assessments including selfmanagement and risk reduction engagement, anxiety reduction and quality of life (Figure 3). However, longterm efficacy outcomes such as mortality, emergency presentations, and readmissions has not been fully assessed with one study reporting failure of nursing-led clinics in this regard. ${ }^{26}$ Nonetheless, further larger studies are warranted to evaluate and validate the efficacy of nurse-led clinics or enhanced nursing role in the post-PCI management with a focus on long term outcomes. The greater nursing role should include all these aspects such as patient education about self-reliance, engagements in risk reduction activities, medicine adherence, scheduled follow-up at secondary cardiac clinics, and professional psychological intervention. These steps can further improve the quality of life, patient's satisfaction, reduce hospital burden with readmissions and may lead into lower mortality.

In conclusion, Nursing-led intervention seems to improve various aspects of post-PCI problems such as self-management, healthy-heart lifestyle modifications, psychological well-being and quality of life. Further research is warranted to develop a structured post-PCI management plan with enhanced responsibilities assigned to nursing staff in order to improve patients' quality of life and satisfaction in the post-discharge period.

\section{Author Contributions}

All authors contributed to data analysis, drafting or revising the article, gave final approval of the version to be 


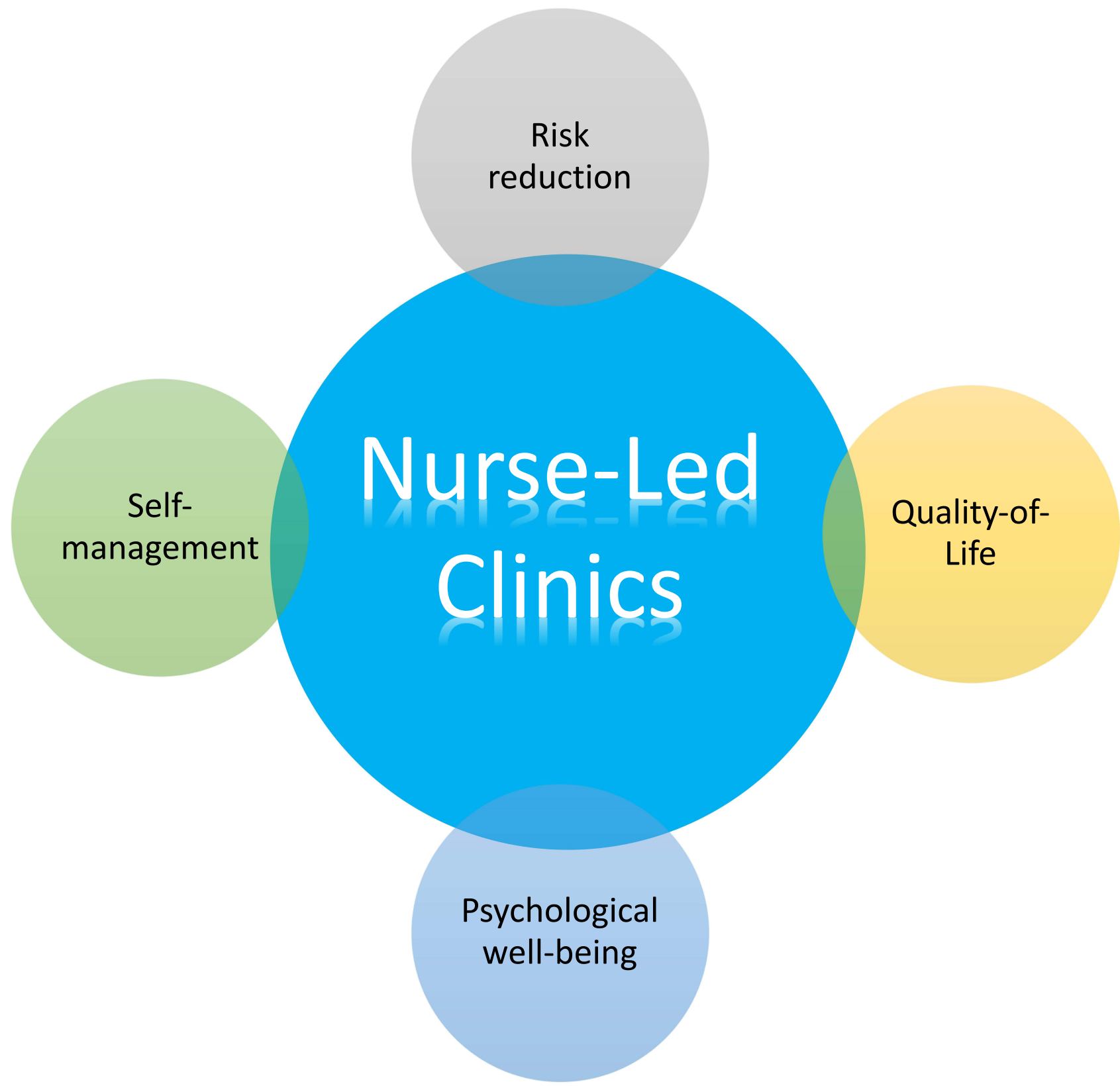

Figure 3 Nursing-led clinics has shown improvement in three main dimensions such as, risk reduction, self-management, psychological improvement, and quality of life.

published, agreed to the submitted journal, and agree to be accountable for all aspects of the work.

\section{Funding}

There is no funding to report.

\section{Disclosure}

The authors report no conflicts of interest in this work.

\section{References}

1. Virani SS, Alonso A, Benjamin EJ, et al. Heart disease and stroke statistics-2020 update: a report from the American Heart Association. Circulation. 2020;141(9):e139-e596. doi:10.1161/cir.000000000000 0757

2. Roth GA, Johnson C, Abajobir A, et al. Global, regional, and national burden of cardiovascular diseases for 10 causes, 1990 to 2015. $\mathrm{J} \mathrm{Am}$ Coll Cardiol. 2017;70(1):1-25. doi:10.1016/j.jacc.2017.04.052

3. Lusis AJ. Atherosclerosis. Nature. 2000;407(6801):233-241. doi: $10.1038 / 35025203$ 
4. Moran AE, Forouzanfar MH, Roth GA, et al. Temporal trends in ischemic heart disease mortality in 21 world regions, 1980 to 2010 : the global burden of disease 2010 study. Circulation. 2014;129 (14):1483-1492. doi:10.1161/circulationaha.113.004042

5. Sampasa-Kanyinga H, Lewis RF. Frequent use of social networking sites is associated with poor psychological functioning among children and adolescents. Cyberpsychol Behav Soc Netw. 2015;18 (7):380-385. doi:10.1089/cyber.2015.0055

6. UN. World population prospects 2019: highlights; 2019. Available from: https://population.un.org/wpp/Publications/Files/WPP2019_ 10KeyFindings.pdf. Accessed October 12, 2021.

7. Institute for Health Metrics and Evaluation (IHME). GBD compare; 2019. Available from: http://vizhub.healthdata.org/gbd-compare. Accessed October 12, 2021.

8. Kandaswamy E, Zuo L. Recent advances in treatment of coronary artery disease: role of science and technology. Int J Mol Sci. 2018;19 (2):424. doi:10.3390/ijms19020424

9. Jia S, Liu Y, Yuan J. Evidence in guidelines for treatment of coronary artery disease. Adv Exp Med Biol. 2020;1177:37-73. doi:10.1007/ 978-981-15-2517-9_2

10. Figulla HR, Lauten A, Maier LS, Sechtem U, Silber S, Thiele H. Percutaneous coronary intervention in stable coronary heart disease is less more? Dtsch Arztebl Int. 2020;117(9):137-144. doi:10.3238/ arztebl.2020.0137

11. Pursnani S, Korley F, Gopaul R, et al. Percutaneous coronary intervention versus optimal medical therapy in stable coronary artery disease. Circ Cardiovasc Interv. 2012;5(4):476-490. doi:10.1161/ CIRCINTERVENTIONS.112.970954

12. Moran AE, Forouzanfar MH, Roth GA, et al. The global burden of ischemic heart disease in 1990 and 2010. Circulation. 2014;129 (14):1493-1501. doi:10.1161/CIRCULATIONAHA.113.004046

13. Pinaire J, Azé J, Bringay S, Cayla G, Landais P. Hospital burden of coronary artery disease: trends of myocardial infarction and/or percutaneous coronary interventions in France 2009-2014. PLoS One. 2019;14(5):e0215649-e. doi:10.1371/journal.pone.0215649

14. Bundhun PK, Soogund MZ, Huang WQ. Same day discharge versus overnight stay in the hospital following percutaneous coronary intervention in patients with stable coronary artery disease: a systematic review and meta-analysis of randomized controlled trials. PLoS One. 2017;12(1):e0169807. doi:10.1371/journal.pone.0169807

15. Chen Y, Lin FF, Marshall AP. Patient and family perceptions and experiences of same-day discharge following percutaneous coronary intervention and those kept overnight. Intensive Crit Care Nurs. 2021;62:102947. doi:10.1016/j.iccn.2020.102947

16. Heyde GS, Koch KT, de Winter RJ, et al. Randomized trial comparing same-day discharge with overnight hospital stay after percutaneous coronary intervention: results of the Elective PCI in Outpatient Study (EPOS). Circulation. 2007;115(17):2299-2306. doi:10.1161/ circulationaha.105.591495
17. Chen Y, Marshall AP, Lin F. Lessons learnt from the implementation of same-day discharge after percutaneous coronary intervention. Aust Crit Care. 2019;32(6):458-464. doi:10.1016/j.aucc.2018.11.066

18. Throndson K, Sawatzky JA, Schultz A. Exploring the perceptions and health behaviours of patients following an elective ad-hoc percutaneous coronary intervention: a qualitative study. Can J Cardiovasc Nurs. 2016;26(2):25-32.

19. Valaker I, Norekvål TM, Råholm MB, Nordrehaug JE, Rotevatn $\mathrm{S}$, Fridlund B. Continuity of care after percutaneous coronary intervention: the patient's perspective across secondary and primary care settings. Eur J Cardiovasc Nurs. 2017;16(5):444-452. doi:10.1177/ 1474515117690298

20. Pettersen TR, Fridlund B, Bendz B, et al. Challenges adhering to a medication regimen following first-time percutaneous coronary intervention: a patient perspective. Int J Nurs Stud. 2018;88:16-24. doi:10.1016/j.ijnurstu.2018.07.013

21. Iles-Smith H, Deaton C, Campbell M, Mercer C, McGowan L. The experiences of myocardial infarction patients readmitted within six months of primary percutaneous coronary intervention. J Clin Nurs. 2017;26(21-22):3511-3518. doi:10.1111/jocn.13715

22. Lindsay P, Sherrard H, Adam M, Wicha C. The impact of a nurse-managed clinic for PTCA patient follow-up. Can $J$ Cardiovasc Nurs. 2000;11(1):13e20.

23. Shim JL, Hwang SY. Long-term effects of nurse-led individualized education on middle-aged patients with acute coronary synrome: a quasi-experimental study. BMC Nurs. 2017;16(1):59. doi:10.1186/ s12912-017-0254-y

24. Corones-Watkins KM, Theobald KA, White KM. Outcomes of a randomised pilot trial of a nurse-led clinic for patients after percutaneous coronary intervention. Aust Crit Care. 2019;32(4):285-292. doi:10.1016/j.aucc.2018.06.009

25. Chang Z, Guo AQ, Zhou AX, et al. Nurse-led psychological intervention reduces anxiety symptoms and improves quality of life following percutaneous coronary intervention for stable coronary artery disease. Aust J Rural Health. 2020;28(2):124-131. doi:10.1111/ ajr. 12587

26. Kotowycz MA, Cosman TL, Tartaglia C, Afzal R, Syal RP, Natarajan MK. Safety and feasibility of early hospital discharge in ST-segment elevation myocardial infarction-a prospective and randomized trial in low-risk primary percutaneous coronary intervention patients (the Safe-Depart Trial). Am Heart J. 2010;159(1):117.e1-6. doi:10.1016/j.ahj.2009.10.024
International Journal of General Medicine

\section{Publish your work in this journal}

The International Journal of General Medicine is an international, peer-reviewed open-access journal that focuses on general and internal medicine, pathogenesis, epidemiology, diagnosis, monitoring and treatment protocols. The journal is characterized by the rapid reporting of reviews, original research and clinical studies across all disease areas. The manuscript management system is completely online and includes a very quick and fair peer-review system, which is all easy to use. Visit http://www.dovepress.com/ testimonials.php to read real quotes from published authors. 